\title{
Does neoadjuvant chemotherapy cancel out the negative survival impact induced by surgical complications after gastrectomy?
}

\author{
Masato Hayashi ${ }^{1} \cdot$ Takaki Yoshikawa $^{1}$ (i) $\cdot$ Masahiro Yura ${ }^{1} \cdot$ Sho Otsuki ${ }^{1} \cdot$ Yukinori Yamagata $^{1} \cdot$ Shinji Morita $^{1}$. \\ Hitoshi Katai ${ }^{1} \cdot$ Toshirou Nishida $^{1}$
}

Received: 8 November 2018 / Accepted: 23 March 2019 / Published online: 3 April 2019

(c) The International Gastric Cancer Association and The Japanese Gastric Cancer Association 2019

\begin{abstract}
Background Postoperative infectious complications (ICs) are associated with a poor prognosis following gastric cancer surgery. Neoadjuvant chemotherapy (NAC) targeting scirrhous-type or bulky nodal disease reportedly exerts a prophylactic effect on the negative impact of ICs. However, a recent study clearly showed that NAC for scirrhous-type disease had no survival benefit. We investigated this prophylactic effect and significant interactions among subgroups of histological response, macroscopic type, and bulky nodal disease.

Methods We examined 115 patients who received NAC followed by radical gastrectomy between January 2008 and December 2015. The overall survival (OS) and disease-free survival (DFS) were compared between those with and without ICs. Our cohort included 62 with type 4/giant type 3, 44 with bulky nodal disease/para-aortic nodal disease, and 25 with other diseases. Results A histological response was observed in 80 patients (69.5\%). Thirty three (28.7\%) developed ICs. There was no significant difference in the OS [hazard ratio (HR) 0.96; 95\% confidence interval (CI) $0.47-1.99, p=0.920$ ] or DFS (HR $0.74 ; 95 \%$ CI $0.40-1.38, p=0.342$ ) by the presence of ICs. The HR was 1.00 in patients who had no response to NAC (grade $0 / 1 \mathrm{a}$ ) and 0.95 in those who responded to NAC (grade 1b/2/3). No subgroups showed significant interactions for the OS.

Conclusions NAC may cancel out the negative impact of morbidity on the survival in advanced gastric cancer patients. The prophylactic effects by NAC do not depend on the tumor type or histological response.
\end{abstract}

Keywords Gastric cancer · Neoadjuvant chemotherapy · Infectious complication

\section{Introduction}

Gastric cancer (GC) is the world's fourth-most common neoplasm and the second leading cause of cancer death [1]. Macroscopically complete tumor removal is essential for the cure of GC, and gastrectomy with lymph node dissection is the most powerful treatment. However, the prognosis of patients with advanced GC remains poor. Recurrence can occur at the peritoneum, liver or distant organs even after curative surgery, suggesting that distant micrometastases were already present $[2,3]$.

Recent studies have shown that postoperative infectious complications (ICs) are associated with a poor prognosis in various types of malignancies, including GC [4-9]. Several

Takaki Yoshikawa

tayoshik@ncc.go.jp

1 Department of Gastric Surgery, National Cancer Center Hospital, 5-1-1 Tsukiji, Chuo-ku, Tokyo 104-0045, Japan investigators have suggested that ICs trigger the release of systemic cytokines during inflammatory response, leaving the growth of the residual cancer cells existing outside of the surgical field [10-12]. Surgeons' efforts to reduce ICs are essential for improving the survival; however, ICs are unavoidable.

Another approach might be neoadjuvant chemotherapy (NAC) before surgery [13-17]. NAC has the potential to eradicate distant micrometastasis, which might cancel out the negative prognostic impact of ICs. Recently, Eto et al. reported that there was no marked difference in the survival between patients with complications and those without complications in 101 patients who received NAC [18]. Although their report was the first to show a potential prophylactic effect of NAC, their study was limited to special types, such as type 4 , giant type 3 , and bulky nodal disease including para-aortic nodal metastases. Furthermore, the median follow-up period of 33.7 months was relatively short for obtaining definitive conclusions. Very recently, the JCOG0501 
phase III study comparing NAC followed by surgery with S-1 and surgery with S-1 could not show superiority of NAC, suggesting that NAC has no effect for eradicating micrometastasis in type 4 or giant type 3 tumors [19]. Without the elimination of micrometastasis by NAC, attractive story of prophylactic effect by NAC might be doubtful.

Given these previous efforts, we conducted the present study to examine whether or not NAC exerts a prophylactic effect against the negative prognostic impact induced by ICs and evaluated the presence of significant interactions in the subgroups of histological response, macroscopic type, and bulky nodal disease.

\section{Methods}

\section{Patients}

The patients were selected from the medical records of consecutive patients who underwent gastrectomy for advanced GC at National Cancer Center Hospital, Tokyo, Japan, from January 2008 to December 2015. The patients met the following inclusion criteria: (1) histologically proven primary gastric adenocarcinoma; and (2) received NAC followed by curative gastrectomy with lymph node dissection. The patients who had undergone $R 2$ or $R 1$ resection were excluded.

Because NAC is not a standard treatment in Japan, the patients who were treated with NAC were basically entered into the clinical trial or followed to the protocol of the trial. The patient population consisted of three groups: (1) cT3-cT4 and any $\mathrm{N}$ following the protocol of the in-house feasibility study of S-1 with oxaliplatin (UMIN-ID: 000007589), (2) extensive nodal swelling along the major branched arteries or para-aortic lymph node swelling (bulky- $N$ ) following the protocol of JCOG0405 (UMIN-ID: C000000094) or JCOG1002 (UMIN-ID: 000006069), and (3) type 4 and giant type $3 \mathrm{GC}$ following the protocol of JCOG0501 (UMIN-ID: C000000279). The extent of nodal dissection was D2 for the in-house study and JCOG 0501 and D2 plus para-aortic nodal dissection for JCOG0405. The extent of tumor spread was evaluated using the 7th edition of the tumor-node-metastasis (TNM) classification established by the Union for International Cancer Control. The histological efficacy of NAC in the primary tumor was evaluated by the histopathological criteria of the Japanese Classification of Gastric Carcinoma (JCGC) [20]. In brief, the histological response was categorized as follows: grade 0 , no effect; grade 1a, viable tumor cells occupy more than $2 / 3$ of the tumorous area (very slight effect); grade $1 \mathrm{~b}$, viable tumor cells remain in more than $1 / 3$ but less than $2 / 3$ of the tumorous area (slight effect); grade 2 , viable tumor cells remain in less than $1 / 3$ of the tumorous area (considerable effect); and grade 3, no viable tumor cells remain (complete response). Patients whose histological response grade 0 and $1 \mathrm{a}$ were defined as non-responders, while whose grade $1 \mathrm{~b}$ or higher were defined as responders.

\section{The definition and evaluation of surgical complications}

According to the Clavien-Dindo classification [21, 22], postoperative ICs were defined as those which were grade IIIa or higher that occurred during hospitalization within 30 days after surgery. The patients were divided into two groups: those with postoperative ICs (IC group) and those without postoperative ICs (NIC group).

Among the complications, we could review during the hospitalization, pancreatic fistula, anastomotic leakage, abdominal abscess, duodenal stump fistula, and pneumonia were defined as infectious complications.

\section{Statistical analyses}

The overall survival (OS) was defined as the period between the date of surgery and death, and the disease-free survival (DFS) was defined as the period from the date of surgery to the first occurrence of any of the following events, including recurrence, distant metastasis, or death from any cause. The data of patients who had not experienced an event were censored at the date of the final observation. The OS and DFS curves were calculated using the Kaplan-Meier method and compared by the log-rank test. The Cox proportional hazards model was used for the uni- and multivariate survival analyses to identify prognosticators. We conducted a multivariate analysis of the OS and DFS using factors that values were $<0.05$ in the univariate analysis (ypT and ypN factors). Patients with missing covariate values were excluded. The Mann-Whitney $U$ test or Chi-squared method was used to compare the two groups. $p$ values $<0.05$ were considered to indicate statistical significance. The survival data were obtained from hospital records. All statistical analyses were performed using the IBM SPSS Statistics software program, version 24 for Windows (Chicago, IL, USA).

\section{Ethics}

The present study was conducted in compliance with the ethical guidelines for clinical research. This study was approved by the Institutional Review Board (IRB) of the National Cancer Center Hospital. 


\section{Results}

\section{Patient characteristics}

A total of 115 patients were included in the present study. ICs were found in 33 patients (28.7\%). Table 1 summarizes the perioperative characteristics before surgery. Sixty-two patients had macroscopic type 4 or giant type 3, 44 had bulky nodal disease or para-aortic nodal metastases, and 35 had others (neither type 4/giant type 3 nor bulky nodal disease/para-aortic nodal metastases). The histological efficacy against the primary tumor was $2(1.7 \%)$ in grade $0,33(28.7 \%)$ in grade $1 \mathrm{a}, 30(26.1 \%)$ in grade $1 \mathrm{~b}, 47$ $(40.9 \%)$ in grade 2 , and $3(2.6 \%)$ in grade 3 . The more than grade $1 \mathrm{~b}$ histological response was $80(69.6 \%)$ in this cohort. This response was achieved in $46(57.5 \%)$ by $\mathrm{S}-1 /$ cisplatin, $13(16.3 \%)$ by S-1/oxaliplatin, $12(15.0 \%)$ by S-1/docetaxel/cisplatin, and $9(11.3 \%)$ by other regimens.

The proportion of men was significantly greater than that of women, and an upper location tended to be more frequent in the IC group than in the NIC group. Furthermore, the operation time was significantly longer and blood loss significantly greater in the IC group than in the NIC group. And postoperative highest CRP level of IC group was significantly higher than that of NIC group.

\section{Surgical morbidity and mortality}

The details of the complications in all patients are shown in Table 2. Pancreatic fistula was the most frequent morbidity ( 21 cases, $18.3 \%$ ), followed by anastomotic leakage ( 4 cases, $3.5 \%$ ) and abdominal abscess (3 cases, 2.6\%). Complications of grade III were found in 47 cases (40.9\%), while those of grade IV or V occurred in 3 cases (2.6\%). Surgical mortality was observed in 1 case $(0.9 \%)$.

\section{OS and DFS analyses}

The OS curve of the IC group was similar to that of the NIC group ( $p=0.920$, Fig. 1a). The 1-year OS was $90.9 \%$ in the IC group and $93.9 \%$ in the NIC group, while the 3 -year OS was $75.8 \%$ in the IC group and $74.4 \%$ in the NIC group. In the univariate Cox analysis, ypT factor and ypN factor only showed $p$ value $<0.05$ (Table 3 ). The hazard ratio (HR) of the IC group against the NIC group and the $p$ value of ICs were 0.96 and 0.920 , respectively, in the univariate analysis. Postoperative highest CRP level did not reach significant difference, either. The multivariate analysis showed that only ypT and ypN were significant independent risk factors for the OS (ypT; $p=0.048$, ypN; $p=0.010)$ (Table 3).
The DFS curves nearly crossed until 12 months and then slightly separated. The DFS was slightly longer in the NIC group than in the IC group, although the difference was not significant (Fig. 1b). In the univariate analysis, ypT factor and ypN factor were significantly associated with the DFS $(p=0.001$, respectively). In the multivariate analysis, for the DFS, both factors remained significant independent risk factors (ypT; $p=0.007, \mathrm{ypN} ; p=0.001$ ) (Table 4).

Then, the survival was compared between the IC and NIC groups stratified by the histological response (Fig. 1c, d). In the responder, the 3-year OS was $63.6 \%$ in the IC group and $63.8 \%$ in the NIC group, which was not significantly different $(p$ value $=0.904)$. In the non-responder, the 3-year OS was $54.5 \%$ in the IC group and $66.7 \%$ in the NIC group, which was also not significantly different $(p$ value $=0.996$ )

\section{Reasons for the death and recurrence patterns}

The median follow-up time for the 115 patients was 54 months. During the follow-up period, 37 patients (32.2\%) died. Thirty-four patients $(29.6 \%)$ died of GC, while 3 (2.6\%) died of other causes. Fifty-five patients $(47.8 \%)$ developed recurrence. Seventeen patients (14.8\%) developed lymph node recurrence, 25 (21.7\%) developed peritoneal recurrence, 1 developed anastomotic recurrence $(0.9 \%)$, and $14(12.2 \%)$ had other site recurrence (7 liver metastasis, 4 lung metastasis, 1 gall bladder metastasis, 1 adrenal metastasis, 1 pleural metastasis). Details are shown in Table 5.

\section{Subgroup analyses}

The results of subgroup analyses of the OS regarding pathological factors (ypT, ypN and histological response grade), macroscopic type, bulky $\mathrm{N}$, and neo or adjuvant chemotherapy are shown in Fig. 2. The HR was 1.00 in patients who had no response to NAC (grade 0/1a) and 0.95 in those who responded to NAC (grade $1 \mathrm{~b} / 2 / 3$ ). There was no significant interaction between the two subgroups $(p=0.658)$. No other subgroups showed clear superiority in the NIC group versus the IC group. There were no interactions showing statistical significance among the subgroups.

\section{Discussion}

We evaluated whether or not NAC exerted prophylactic effects against the negative prognostic impact induced by ICs and assessed its interaction among subgroups in this study. Compared with Eto's report, the strength of this study is its larger cohort size including common types of GC, longer follow-up (more than 3 years), and analyses of interactions among subgroups. The present study showed that the OS was not significantly affected by the occurrence 
Table 1 Perioperative patient characteristics between IC group and NIC group

\begin{tabular}{|c|c|c|c|c|}
\hline Variables & All patients $N=115$ & IC group $N=33(28.7 \%)$ & NIC group $N=82(71.3 \%)$ & $p$ value \\
\hline Age (years) & $63.3( \pm 10.8)$ & $62.6( \pm 11.5)$ & $63.7( \pm 10.5)$ & 0.359 \\
\hline Gender & & & & 0.042 \\
\hline Male & $81(70.4 \%)$ & $28(84.8 \%)$ & $53(64.6 \%)$ & \\
\hline Female & $34(29.6 \%)$ & $5(15.2 \%)$ & $29(35.4 \%)$ & \\
\hline ASA-PS & & & & 0.544 \\
\hline 1 & $11(9.6 \%)$ & $2(6.1 \%)$ & $9(11.0 \%)$ & \\
\hline 2 & $94(81.7 \%)$ & $27(81.8 \%)$ & $67(81.7 \%)$ & \\
\hline 3 & $10(8.7 \%)$ & $4(12.1 \%)$ & $6(7.3 \%)$ & \\
\hline Tumor location $^{\mathrm{a}}$ & & & & 0.085 \\
\hline Upper & $46(40 \%)$ & $19(57.6 \%)$ & $27(32.9 \%)$ & \\
\hline Middle & $39(33.9 \%)$ & $9(27.3 \%)$ & $30(36.6 \%)$ & \\
\hline Lower & $20(17.4 \%)$ & $4(12.1 \%)$ & $16(19.5 \%)$ & \\
\hline Whole & $10(8.7 \%)$ & $1(3.0 \%)$ & $9(11.0 \%)$ & \\
\hline Bulky N/\#16LN metastasis & $44(38.3 \%)$ & $15(45.5 \%)$ & $29(35.4 \%)$ & 1.000 \\
\hline Macroscopic giant type3/type4 & $62(53.9 \%)$ & $14(42.4 \%)$ & $48(58.5 \%)$ & 0.149 \\
\hline ycStage & & & & 0.143 \\
\hline I & $3(2.6 \%)$ & $0(0 \%)$ & $3(3.7 \%)$ & \\
\hline II & $47(40.9 \%)$ & $10(30.3 \%)$ & $37(45.1 \%)$ & \\
\hline III & $65(56.5 \%)$ & $23(69.7 \%)$ & $42(51.2 \%)$ & \\
\hline NAC regimen & & & & 0.926 \\
\hline S-1 and cisplatin & $74(64.3 \%)$ & $20(60.6 \%)$ & $54(65.9 \%)$ & \\
\hline S-1 and oxaliplatin & $14(12.2 \%)$ & $4(12.1 \%)$ & $10(12.2 \%)$ & \\
\hline S-1, docetaxel and cisplatin & $13(11.3 \%)$ & $4(12.1 \%)$ & $9(11.0 \%)$ & \\
\hline Others & $14(12.2 \%)$ & $5(15.2 \%)$ & $9(11.0 \%)$ & \\
\hline Surgical Procedure & & & & 0.815 \\
\hline Distal gastrectomy & $29(25.2 \%)$ & $7(21.2 \%)$ & $22(26.8 \%)$ & \\
\hline Total gastrectomy & $83(72.3 \%)$ & $25(75.8 \%)$ & $58(70.7 \%)$ & \\
\hline Other & $3(2.6 \%)$ & $1(3.0 \%)$ & $2(2.4 \%)$ & \\
\hline Operation time (min) & $337.1( \pm 111.1)$ & $396.8( \pm 138.1)$ & $313.1( \pm 88.4)$ & 0.001 \\
\hline Blood loss (ml) & $711.5( \pm 627.0)$ & $814.7( \pm 536.6)$ & $670.0( \pm 658.4)$ & 0.039 \\
\hline Extent of lymphadenectomy & & & & 0.806 \\
\hline D2 & $48(41.7 \%)$ & $15(45.5 \%)$ & $33(40.2 \%)$ & \\
\hline $\mathrm{D} 2+$ & $40(34.8 \%)$ & $10(31.3 \%)$ & $30(36.6 \%)$ & \\
\hline D3 & $27(23.5 \%)$ & $8(25.0 \%)$ & $19(23.2 \%)$ & \\
\hline $\mathrm{ypT}^{\mathrm{a}}$ factor & & & & 0.185 \\
\hline 0 & $4(3.5 \%)$ & $0(0 \%)$ & $4(4.9 \%)$ & \\
\hline $\mathrm{T} 1 \mathrm{a}$ & $1(0.9 \%)$ & $0(0 \%)$ & $1(1.2 \%)$ & \\
\hline $\mathrm{T} 1 \mathrm{~b}$ & $13(11.3 \%)$ & $5(15.2 \%)$ & $8(9.8 \%)$ & \\
\hline $\mathrm{T} 2$ & $17(14.8 \%)$ & $7(21.2 \%)$ & $10(12.2 \%)$ & \\
\hline T3 & $51(44.3 \%)$ & $13(39.4 \%)$ & $38(46.3 \%)$ & \\
\hline $\mathrm{T} 4 \mathrm{a}$ & $25(21.7 \%)$ & $5(15.2 \%)$ & $20(24.4 \%)$ & \\
\hline $\mathrm{T} 4 \mathrm{~b}$ & $4(3.5 \%)$ & $3(9.1 \%)$ & $1(1.2 \%)$ & \\
\hline $\mathrm{ypN}^{\mathrm{a}}$ factor & & & & 0.374 \\
\hline 0 & $39(33.9 \%)$ & $14(42.4 \%)$ & $25(30.5 \%)$ & \\
\hline 1 & $24(20.9 \%)$ & $9(27.3 \%)$ & $15(18.3 \%)$ & \\
\hline 2 & $26(22.6 \%)$ & $6(18.2 \%)$ & $20(24.4 \%)$ & \\
\hline 3 & $26(22.6 \%)$ & $4(12.1 \%)$ & $22(26.8 \%)$ & \\
\hline$M$ factor & & & & 0.727 \\
\hline 0 & $104(90.4 \%)$ & $29(87.9 \%)$ & $75(91.5 \%)$ & \\
\hline 1 & $11(9.6 \%)$ & $4(12.1 \%)$ & $7(8.5 \%)$ & \\
\hline
\end{tabular}


Table 1 (continued)

\begin{tabular}{llll}
\hline Variables & All patients $N=115$ & IC group $N=33(28.7 \%)$ & NIC group $N=82(71.3 \%)$ \\
\hline ypStage $^{\mathrm{a}}$ & & & $p$ value \\
0 & $3(2.6 \%)$ & $0(0 \%)$ & $3(3.7 \%)$ \\
I & $19(16.5 \%)$ & $8(24.2 \%)$ & $11(13.4 \%)$ \\
II & $37(32.3 \%)$ & $9(27.3 \%)$ & $28(34.1 \%)$ \\
III & $45(39.1 \%)$ & $12(36.4 \%)$ & $33(40.2 \%)$ \\
IV & $11(9.6 \%)$ & $4(12.1 \%)$ & $7(8.5 \%)$ \\
Postoperative highest CRP level & $16.34( \pm 6.94)$ & $21.9( \pm 7.34)$ & $14.1( \pm 5.34)$ \\
Histological response of primary lesion & & & $1(1.2 \%)$ \\
0 & $2(1.7 \%)$ & $1(3.0 \%)$ & $23(28.0 \%)$ \\
1 la & $33(28.7 \%)$ & $10(30.3 \%)$ & $20(24.4 \%)$ \\
1b & $30(26.1 \%)$ & $10(30.3 \%)$ & $35(42.7 \%)$ \\
2 & $47(40.9 \%)$ & $12(36.4 \%)$ & $3(3.7 \%)$ \\
3 & $3(2.6 \%)$ & $0(0 \%)$ & 0.692 \\
Adjuvant chemotherapy & & & $63(76.8 \%)$ \\
Present & $84(73.0 \%)$ & $21(63.6 \%)$ & $19(23.2 \%)$ \\
Absent & $31(27.0 \%)$ & $12(36.4 \%)$ & $7.89( \pm 7.89)$ \\
Duration of adjuvant chemotherapy (months) & $7.18( \pm 7.46)$ & $6.21( \pm 6.28)$ & 0.168 \\
\hline
\end{tabular}

${ }^{a}$ According to the seventh edition of the International Union Against

Cancer tumor, node, metastasis (TNM) classification system

Table 2 Details of all complications

\begin{tabular}{lcclccc}
\hline Complications & \multicolumn{2}{c}{ Grade according to Clavien Dindo classification } & \multicolumn{2}{c}{ Total (\%) } \\
\cline { 2 - 5 } & IIIa & IIIb & IVa & IVb & V & \\
\hline Pancreatic fistula & 21 & 0 & 0 & 0 & 0 & $21(18.3 \%)$ \\
Anastomitic leakage & 2 & 2 & 0 & 0 & 0 & $4(3.5 \%)$ \\
Abdominal abscess & 3 & 0 & 0 & 0 & 0 & $3(2.6 \%)$ \\
Duodenal stump fistula & 1 & 1 & 0 & 0 & 1 & $3(2.6 \%)$ \\
Pneumonia & 1 & 0 & 0 & 0 & 0 & $1(0.9 \%)$ \\
Bleeding & 3 & 1 & 0 & 0 & 0 & $4(3.5 \%)$ \\
Lymph fistula & 1 & 0 & 0 & 0 & 0 & $1(0.9 \%)$ \\
Acute kidney failure & 0 & 0 & 1 & 0 & 0 & $1(0.9 \%)$ \\
Arrithmia & 0 & 0 & 1 & 0 & 0 & $1(0.9 \%)$ \\
Others & 7 & 1 & 0 & 0 & 0 & $8(7.0 \%)$ \\
Total & 39 & 5 & 2 & 0 & 1 & $47(40.9 \%)$ \\
\hline
\end{tabular}

of postoperative ICs, which was supported by a similar trend being noted in the DFS and a similar recurrence between the ICs and the NICs. Furthermore, there was no clear trend toward a better survival in the NIC group than in the IC group among subgroups of histological response or specific types of type 4 or bulky nodal disease. These results strengthen the hypothesis that NAC cancels out the poor prognosis induced by postoperative ICs after gastrectomy. Prophylactic effects of NAC against the negative prognostic impact of morbidity do not appear to depend on the histological response or type of tumors.

There was no significant interaction between the responders and the non-responders. Moreover, the survival curves were almost similar between the IC and the NIC groups regardless of the histological response. These results suggested that the histological response did not affect the prophylactic effect of NAC. Previously, Eto et al. claimed in their report that the elimination of micrometastases by NAC contributed to the abolition of the negative prognostic impact of ICs after gastrectomy [18]. However, it is unclear whether NAC really eradicates micrometastasis. It would be also interesting to see relationships between miscrometastasis and response to NAC. Further study must be focused on these issues.

The HR in this study population was almost 1.0 regardless of the histological response. Histological response 


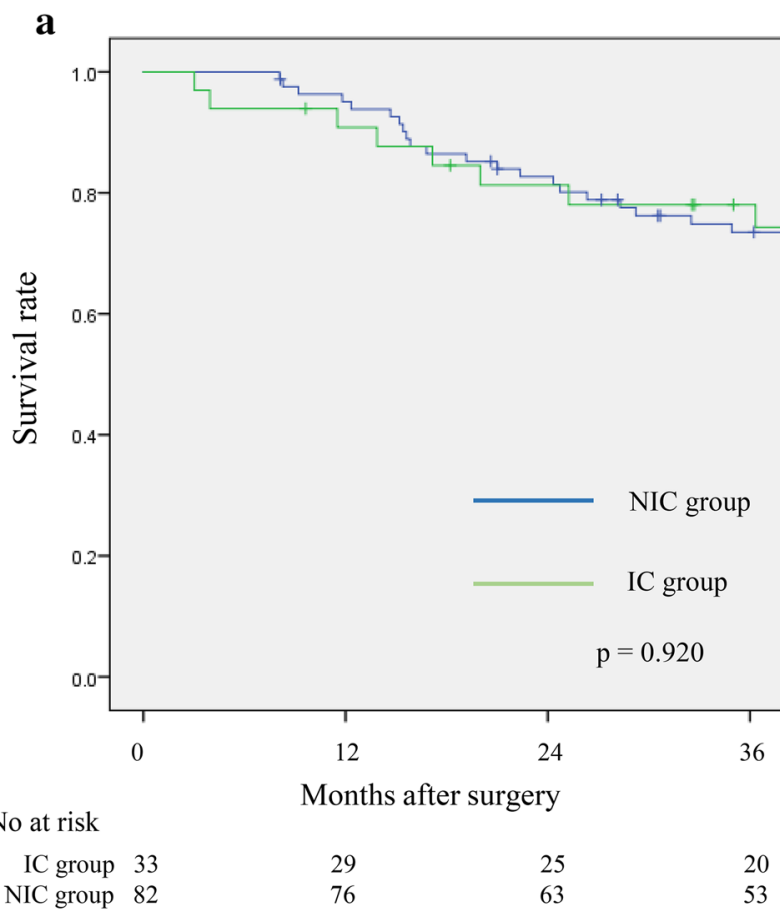

Kaplan-Meier analysis of overall survival between IC group and NIC group

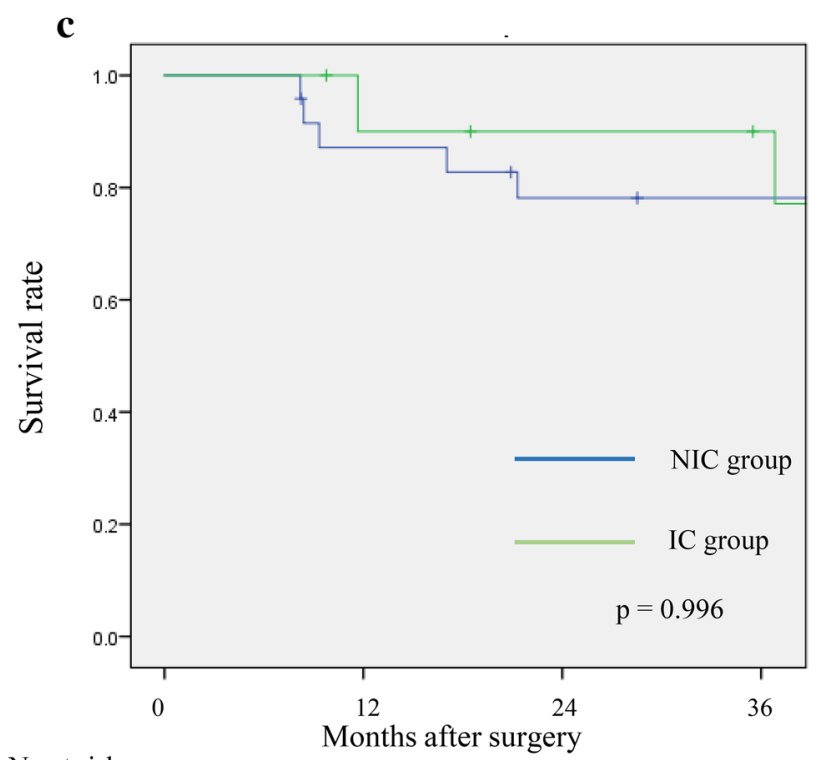

No at risk

IC group 11

NIC group 24

9

20

8

6

Kaplan-Meier analysis of overall survival

between IC group and NIC group in non-responders b

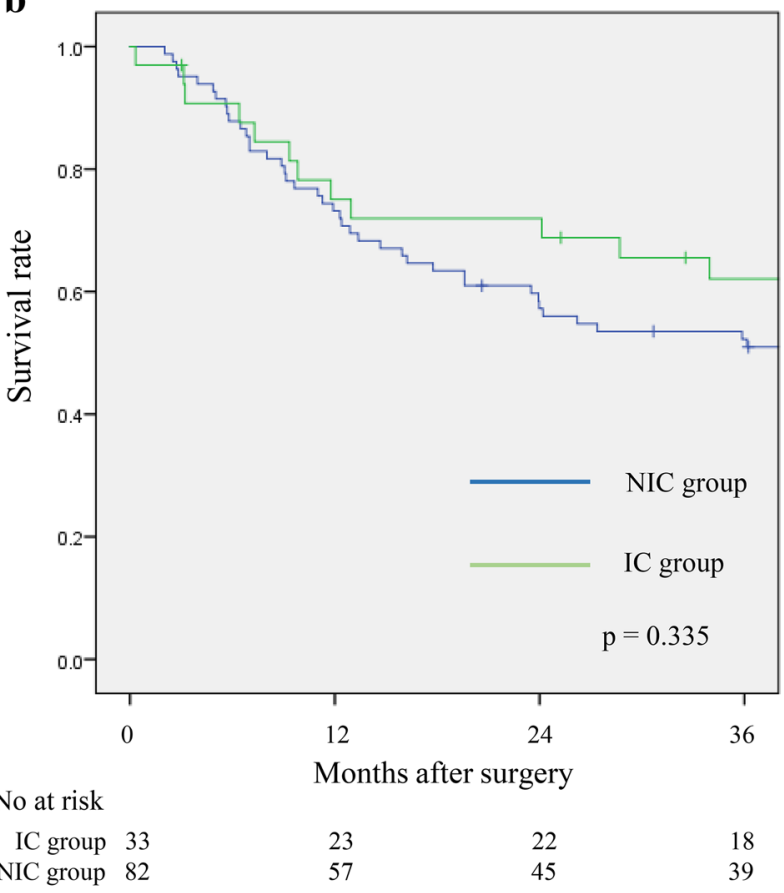

Kaplan-Meier analysis of disease free survival between IC group and NIC group

d

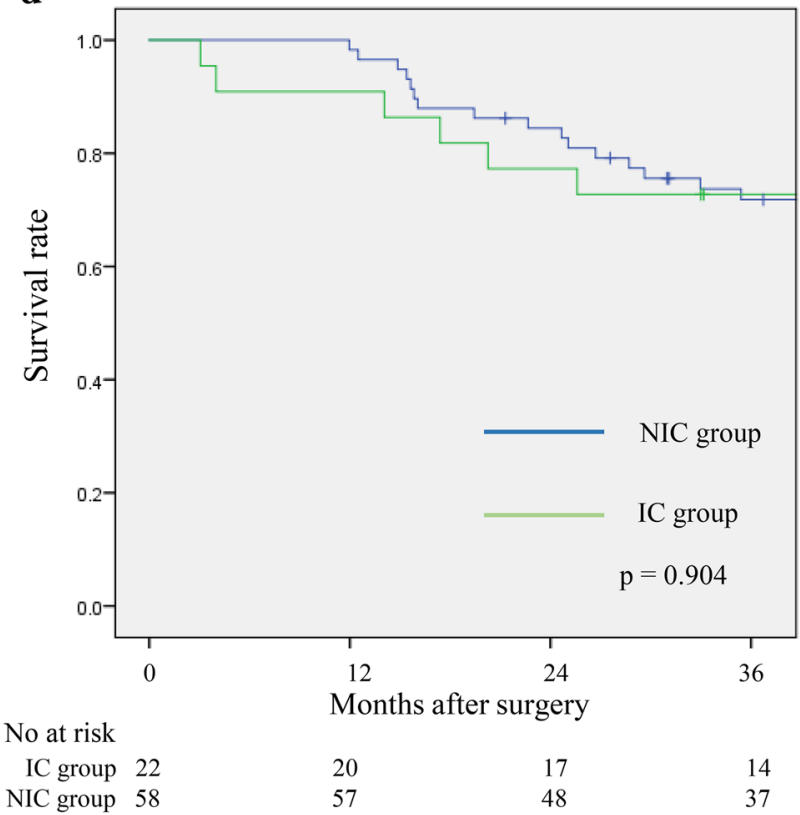

Kaplan-Meier analysis of overall survival between IC group and NIC group in responders

Fig. 1 A Kaplan-Meier analysis of the survival between the IC group and the NIC group. a Overall survival (OS) and (b) disease-free survival (DFS) in the whole cohort and (c) OS in the non-responders and (d) OS in the responders

was a good surrogate endpoint for the survival, which was shown especially in the western cohort [23]. Different from the western countries where NAC was introduced as the standard treatment, there are few reliable studies showing that good histological response was associated with better survival in Japan, because NAC is not a standard treatment 
Table 3 Univariate and multivariate Cox proportional hazards analyses of the clinicopathological factors for the overall survival

\begin{tabular}{|c|c|c|c|c|c|c|c|}
\hline \multirow[t]{2}{*}{ Factors } & \multirow[t]{2}{*}{ Number of patients (\%) } & \multicolumn{3}{|c|}{ Univariate } & \multicolumn{3}{|c|}{ Multivariate } \\
\hline & & HR & $95 \% \mathrm{CI}$ & $p$ value & HR & $95 \% \mathrm{CI}$ & $p$ value \\
\hline Age (years) & & & & 0.973 & & & \\
\hline$\geq 63$ & $69(60 \%)$ & 1.00 & & & & & \\
\hline$<63$ & $46(40 \%)$ & 0.94 & $0.51-1.87$ & & & & \\
\hline Gender & & & & 0.800 & & & \\
\hline Male & $81(70.4 \%)$ & 1.00 & & & & & \\
\hline Female & $34(29.6 \%)$ & 1.10 & $0.54-2.22$ & & & & \\
\hline ASA-PS & & & & 0.276 & & & \\
\hline 1 & $11(9.6 \%)$ & 1.00 & & & & & \\
\hline $2 / 3$ & $104(90.4 \%)$ & 2.23 & $0.53-9.38$ & & & & \\
\hline Operation time (min) & & & & 0.422 & & & \\
\hline$<337$ & $65(56.5 \%)$ & 1.00 & & & & & \\
\hline$\geq 337$ & $50(43.5 \%)$ & 1.30 & $0.68-2.49$ & & & & \\
\hline Blood loss (ml) & & & & 0.283 & & & \\
\hline$<712$ & $74(64.3 \%)$ & 1.00 & & & & & \\
\hline$\geq 712$ & $41(35.7 \%)$ & 1.53 & $0.70-3.35$ & & & & \\
\hline Extent of lymphadenectomy & & & & 0.070 & & & \\
\hline D2/D2+ & $88(76.5 \%)$ & 1.00 & & & & & \\
\hline D3 & $27(23.5 \%)$ & 1.89 & $0.95-3.77$ & & & & \\
\hline $\mathrm{ypT}^{\mathrm{a}}$ factor & & & & 0.013 & & & 0.048 \\
\hline 0/T1a/T1b/T2 & $35(30.4 \%)$ & 1.00 & & & 1.00 & & \\
\hline $\mathrm{T} 3 / \mathrm{T} 4 \mathrm{a} / \mathrm{T} 4 \mathrm{~b}$ & $80(69.6 \%)$ & 3.30 & $1.29-8.49$ & & 2.62 & $1.01-6.79$ & \\
\hline $\mathrm{ypN}^{\mathrm{a}}$ factor & & & & 0.003 & & & 0.010 \\
\hline 0 & $39(33.9 \%)$ & 1.00 & & & 1.00 & & \\
\hline $1 / 2 / 3$ & $76(66.1 \%)$ & 4.12 & $1.60-10.57$ & & 3.47 & $1.34-9.00$ & \\
\hline Histological response of primary lesion & & & & 0.766 & & & \\
\hline $0 / 1 \mathrm{a}$ & $35(30.4 \%)$ & 1.00 & & & & & \\
\hline $1 b / 2 / 3$ & $80(69.6 \%)$ & 0.90 & $0.43-1.85$ & & & & \\
\hline Adjuvant chemotherapy & & & & 0.268 & & & \\
\hline Absent & $31(27.0 \%)$ & 1.00 & & & & & \\
\hline Present & $84(73.0 \%)$ & 1.59 & $0.70-3.63$ & & & & \\
\hline Inflammatory complication grade IIIa or higher & & & & 0.920 & & & \\
\hline Absent & $82(71.3 \%)$ & 1.00 & & & & & \\
\hline Present & $33(28.7 \%)$ & 0.96 & $0.47-1.99$ & & & & \\
\hline Postoperative highest CRP level & & & & 0.318 & & & \\
\hline$<15.5$ & $59(51.3 \%)$ & 1.00 & & & & & \\
\hline$\geq 15.5$ & $56(48.7 \%)$ & 1.39 & $0.72-2.67$ & & & & \\
\hline Macroscopic giant type $3 /$ type 4 & & & & 0.076 & & & \\
\hline Absent & $53(46.1 \%)$ & 1.00 & & & & & \\
\hline Present & $62(53.9 \%)$ & 1.85 & $0.94-3.63$ & & & & \\
\hline NAC regimen & & & & 0.345 & & & \\
\hline S-1 and cisplatine & $74(64.3 \%)$ & 1.00 & & & & & \\
\hline S-1 and oxaliplatin & $14(12.2 \%)$ & 0.97 & $0.46-2.07$ & & & & \\
\hline Others & $27(23.5 \%)$ & 0.34 & $0.07-1.57$ & & & & \\
\hline
\end{tabular}

${ }^{\mathrm{a}}$ According to the seventh edition of the International Union Against Cancer tumor, node, metastasis (TNM) classification system

in Japan. There was only one reliable study to examine this topic using prospective study. Nakamura et al. examined surrogacy of histological response in 188 patients who enrolled in several JCOG phase II studies of NAC targeting type 3/giant type 3 (JCOG0002 and JCOG0210) and bulky nodal disease (JCOG0001 and JCOG0405) [24]. 
Table 4 Univariate and multivariate Cox proportional hazards analyses of the clinicopathological factors for the disease-free survival

\begin{tabular}{|c|c|c|c|c|c|c|c|}
\hline \multirow[t]{2}{*}{ Factors } & \multirow[t]{2}{*}{ Number of patients (\%) } & \multicolumn{3}{|c|}{ Univariate } & \multicolumn{3}{|c|}{ Multivariate } \\
\hline & & HR & $95 \% \mathrm{CI}$ & $p$ value & HR & $95 \% \mathrm{CI}$ & $p$ value \\
\hline Age (years) & & & & 0.647 & & & \\
\hline$\geq 63$ & $69(60 \%)$ & 1.00 & & & & & \\
\hline$<63$ & $46(40 \%)$ & 1.14 & $0.66-1.96$ & & & & \\
\hline Gender & & & & 0.468 & & & \\
\hline Male & $81(70.4 \%)$ & 1.00 & & & & & \\
\hline Female & $34(29.6 \%)$ & 1.24 & $0.68-2.25$ & & & & \\
\hline ASA-PS & & & & 0.073 & & & \\
\hline 1 & $11(9.6 \%)$ & 1.00 & & & & & \\
\hline $2 / 3$ & $104(90.4 \%)$ & 3.64 & $0.87-14.94$ & & & & \\
\hline Operation time (min) & & & & 0.319 & & & \\
\hline$<337$ & $65(56.5 \%)$ & 1.00 & & & & & \\
\hline$\geq 337$ & $50(43.5 \%)$ & 1.31 & $0.77-2.22$ & & & & \\
\hline Blood loss (ml) & & & & 0.195 & & & \\
\hline$<712$ & $74(64.3 \%)$ & 1.00 & & & & & \\
\hline$\geq 712$ & $41(35.7 \%)$ & 1.43 & $0.83-2.45$ & & & & \\
\hline Extent of lymphadenectomy & & & & 0.136 & & & \\
\hline $\mathrm{D} 2 / \mathrm{D} 2+$ & $88(76.5 \%)$ & 1.00 & & & & & \\
\hline D3 & $27(23.5 \%)$ & 1.57 & $0.87-2.85$ & & & & \\
\hline $\mathrm{ypT}^{\mathrm{a}}$ factor & & & & 0.001 & & & 0.007 \\
\hline 0/T1a/T1b/T2 & $35(30.4 \%)$ & 1.00 & & & 1.00 & & \\
\hline $\mathrm{T} 3 / \mathrm{T} 4 \mathrm{a} / \mathrm{T} 4 \mathrm{~b}$ & $80(69.6 \%)$ & 3.40 & $1.60-7.20$ & & 2.81 & $1.32-6.00$ & \\
\hline $\mathrm{ypN}^{\mathrm{a}}$ factor & & & & 0.001 & & & 0.001 \\
\hline 0 & $39(33.9 \%)$ & 1.00 & & & 1.00 & & \\
\hline $1 / 2 / 3$ & $76(66.1 \%)$ & 3.81 & $1.86-7.80$ & & 3.28 & $1.59-6.74$ & \\
\hline Histological response of primary lesion & & & & 0.644 & & & \\
\hline $0 / 1 \mathrm{a}$ & $35(30.4 \%)$ & 1.00 & & & & & \\
\hline $1 b / 2 / 3$ & $80(69.6 \%)$ & 1.14 & $0.65-2.00$ & & & & \\
\hline Adjuvant chemotherapy & & & & 0.131 & & & \\
\hline Absent & $31(27.0 \%)$ & 1.00 & & & & & \\
\hline Present & $84(73.0 \%)$ & 1.70 & $0.85-3.37$ & & & & \\
\hline Inflammatory complication grade IIIa or higher & & & & 0.342 & & & \\
\hline Absent & $82(71.3 \%)$ & 1.00 & & & & & \\
\hline Present & $33(28.7 \%)$ & 0.74 & $0.40-1.38$ & & & & \\
\hline Postoperative highest CRP level & & & & 0.501 & & & \\
\hline$<15.5$ & $59(51.3 \%)$ & 1.00 & & & & & \\
\hline$\geq 15.5$ & $56(48.7 \%)$ & 1.20 & $0.71-2.04$ & & & & \\
\hline Macroscopic giant type $3 /$ type 4 & & & & 0.076 & & & \\
\hline Absent & $53(46.1 \%)$ & 1.00 & & & & & \\
\hline Present & $62(53.9 \%)$ & 1.64 & $0.95-2.82$ & & & & \\
\hline NAC regimen & & & & 0.134 & & & \\
\hline S-1 and cisplatine & $74(64.3 \%)$ & 1.00 & & & & & \\
\hline S-1 and oxaliplatin & $14(12.2 \%)$ & 1.22 & $0.64-2.32$ & & & & \\
\hline Others & $27(23.5 \%)$ & 0.37 & $0.11-1.32$ & & & & \\
\hline
\end{tabular}

${ }^{\mathrm{a}}$ According to the seventh edition of the International Union Against Cancer tumor, node, metastasis (TNM) classification system

Relationship between good histological response and better survival was demonstrated in phase II studies targeting bulky nodal disease, while that was not demonstrated in the trial targeting type 4/giant type 3 . The phase II trials targeting bulky nodal disease (JCOG0001 and JCOG0405) did not permit any adjuvant chemotherapy after surgery. More 
Table 5 Reasons for recurrence patterns between IC group and NIC group

\begin{tabular}{lcc}
\hline Variables & IC group $N=33$ & NIC group $N=82$ \\
\hline Lymph node recurrence & $4(12.1 \%)$ & $13(15.9 \%)$ \\
Peritoneal recurrence & $6(18.2 \%)$ & $19(23.2 \%)$ \\
Liver metastasis recurrence & $3(9.1 \%)$ & $4(4.9 \%)$ \\
Lung metastasis recurrence & $0(0 \%)$ & $4(4.9 \%)$ \\
Gall bladder metastasis recur- & $0(0 \%)$ & $1(1.2 \%)$ \\
$\quad$ rence & & \\
Adrenal metastasis recurrence & $0(0 \%)$ & $1(1.2 \%)$ \\
Pleural recurrence & $1(3.0 \%)$ & $0(0 \%)$ \\
Anastomotic recurrence & $0(0 \%)$ & $1(1.2 \%)$ \\
Number of recurrence case & $14(42.4 \%)$ & $41(50.0 \%)$ \\
\hline
\end{tabular}

recently, JCOG0501 phase III study reported at ASCO 2018, a first phase III study of NAC in Japan, has revealed that giant type 3 and type 4 advanced gastric cancer did not show prognostic improvement, even if they were treated with NAC [19]. Furthermore, there was no survival difference between primary surgery group and NAC group, though NAC group had $57.9 \%$ response rate. Seventy-seven out of 133 patients had histological response grade $1 \mathrm{~b}$ or higher. Thus, there is no concrete evidence showing that histological response was related with the survival in Japan. It is unclear whether surrogacy of histological response is shown in common type or whether postoperative efficient chemotherapy with S-1 offset the surrogacy. In our cohort, 53.9\% was type 4/giant type 3 and $73 \%$ had received S-1 adjuvant chemotherapy after surgery.

In the present study, no significant interaction was observed between the subgroup of type 4/giant type 3 and other macroscopic types. A significant interaction was also not observed based on the presence of bulky nodal disease. These results suggest that the prophylactic effect did not differ depending on the macroscopic type or presence of bulky nodal disease. In contrast to type 4 or giant type 3 disease, bulky nodal disease is considered a suitable target of NAC. In the JCOG0001 [2] and JCOG0405 [25] phase II trials, an excellent survival was obtained with NAC for bulky nodal disease. Considering these, prophylactic effect of NAC would not be related with efficacy of NAC.

Why then does NAC exert a prophylactic effect against the negative prognostic impact of ICs after gastrectomy? The exact mechanisms are unclear. Our study as well as Eto showed that the incidence of inflammatory reactions after surgery differed between the IC and NIC groups, indicating differences in the release of cytokines from lymphocytes between the groups. The growth of micrometastasis would be enhanced by ICs. Chemotherapy is known to be a potential immunomodulatory agent, and

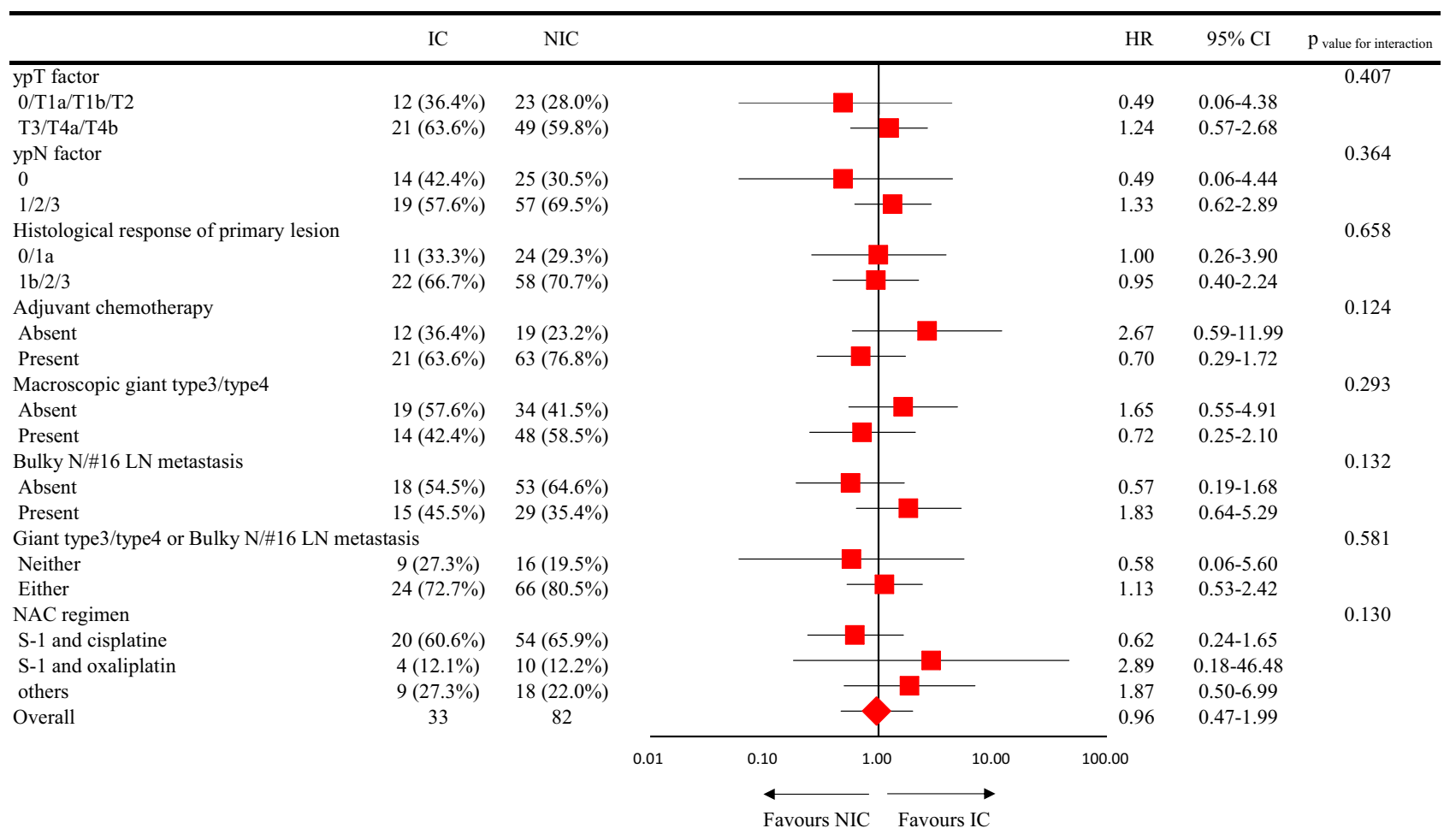

Fig. 2 Subgroup analyses. No subgroup showed clear superiority in the NIC group versus the IC group. No interactions showed statistical significance among the subgroups 
many chemotherapy-mediated stress pathways modulate the expression of NK cell-activating and inhibitory ligands, rendering tumor cells more immunogenic [26]. These mechanisms might be involved in the prophylactic effects of NAC.

There are some limitations associated with the present study. First, this was a retrospective, single-center study. We cannot deny the possibility of several biases related to the retrospective nature of the study. However, the cohort enrolled in the present study is mostly limited to the prospective clinical trial. The selection of patients, treatment strategy, and follow-up was strictly defined by the protocol. Therefore, biases caused by the retrospective nature are likely limited. Second, the sample size was not very large, with each subgroup being particularly small. The reliability of the results of the subgroup analyses might, therefore, be somewhat low. However, the findings of the recent phase III trial JCOG0501 strongly support our hypothesis that the prophylactic effect of NAC was not due to the elimination of micrometastases by this therapy [19]. Third, we have no concurrent control of the patients who received primary surgery and do not know whether IC worsens the survival in the control. However, many previous studies clearly demonstrated that the patients who developed IC had poorer prognosis than those who did not when the patients received primary surgery. Therefore, we considered that NAC had prophylactic effect against negative survival impact induced by surgery. Forth, we could not ignore the effect of adjuvant chemotherapy. In the present study, the patients received adjuvant chemotherapy following each protocol. All protocols described that patients after $R 0$ resection must receive $\mathrm{S}-1$ adjuvant chemotherapy for 1 year except JCOG0405 trial targeting bulky nodal disease. The survival could be affected not only by the presence of absence of adjuvant chemotherapy but also by its duration. However, there was no significant difference in these factors between the IC and the NIC groups in this study. Thus, the effect of adjuvant chemotherapy would be limited.

In conclusion, the present study confirmed the hypothesis that NAC could eliminate the negative impact of postoperative ICs on the OS and DFS in advanced GC patients. The prophylactic effects of NAC against the negative prognostic impact induced by morbidity do not appear to depend on the type of tumor or histological response.

Acknowledgements This study was approved by an Ethical Committee of the National Cancer Center Hospital (No: 2017-077).

\section{Compliance with ethical standards}

Conflict of interest The authors have no conflicts of interest that are directly relevant to the content of this manuscript.

\section{References}

1. Kamangar F, Dores GM, Anderson WF. Patterns of cancer incidence, mortality, and prevalence across five continents: defining priorities to reduce cancer disparities in different geographic regions of the world. J Clin Oncol. 2006;24:2137-50.

2. Yoshikawa T, Sasako M, Yamamoto S, Sano T, Imamura H, Fujitani K, et al. Phase II study of neoadjuvant chemotherapy and extended surgery for locally advanced gastric cancer. Br J Surg. 2009;96:1015-22.

3. Gretschel S, Siegel R, Estévez-Schwarz L, Hünerbein M, Schneider U, Schlag PM, et al. Surgical strategies for gastric cancer with synchronous peritoneal carcinomatosis. Br J Surg. 2006;93:1530-5.

4. Kataoka K, Takeuchi H, Mizusawa J, Igaki H, Ozawa S, Abe $\mathrm{T}$, et al. Prognostic impact of postoperative morbidity after esophagectomy for esophageal cancer: exploratory analysis of JCOG9907. Ann Surg. 2017;265:1152-7.

5. Khuri SF, Henderson WG, DePalma RG, Mosca C, Healey NA, Kumbhani DJ, et al. Determinants of long-term survival after major surgery and the adverse effect of postoperative complications. Ann Surg. 2005;242:326-41.

6. Krarup PM, Nordholm-Carstensen A, Jorgensen LN, Harling $\mathrm{H}$. Anastomotic leak increases distant recurrence and long-term mortality after curative resection for colonic cancer: a nationwide cohort study. Ann Surg. 2014;259:930-8.

7. Sierzega M, Kolodziejczyk P, Kulig J. Impact of anastomotic leakage on long-term survival after total gastrectomy for carcinoma of the stomach. Br J Surg. 2010;97:1035-42.

8. Tokunaga M, Tanizawa Y, Bando E, Kawamura T, Terashima M. Poor survival rate in patients with postoperative intra-abdominal infectious complications following curative gastrectomy for gastric cancer. Ann Surg Oncol. 2013;20:1575-83.

9. Kubota T, Hiki N, Sano T, Nomura S, Nunobe S, Kumagai K, et al. Prognostic significance of complications after curative surgery for gastric cancer. Ann Surg Oncol. 2014;21:891-8.

10. Germano G, Allavena P, Mantovani A. Cytokines as a key component of cancer-related inflammation. Cytokine. 2008;43:374-9.

11. Coussens LM, Werb Z. Inflammation and cancer. Nature. 2002;420:860-7.

12. Matsuda S, Takeuchi H, Kawakubo H, Fukuda K, Nakamura R, Takahashi T, et al. Correlation between intense postoperative inflammatory response and survival of esophageal cancer patients who underwent transthoracic esophagectomy. Ann Surg Oncol. 2015;22(13):4453-60. https://doi.org/10.1245/s10434-015-45575 Epub 2015 Apr 18.

13. Cunningham D, Allum WH, Stenning SP, Thompson JN, Van de Velde CJ, Nicolson M, et al. Perioperative chemotherapy versus surgery alone for resectable gastroesophageal cancer. N Engl J Med. 2006;355:11-20.

14. Schuhmacher C, Gretschel S, Lordick F, Reichardt P, Hohenberger $\mathrm{W}$, Eisenberger $\mathrm{CF}$, et al. Neoadjuvant chemotherapy compared with surgery alone for locally advanced cancer of the stomach and cardia: european organisation for research and treatment of cancer randomized trial 40954. J Clin Oncol. 2010;28:5210-8.

15. Tsuburaya A, Nagata N, Cho H, Hirabayashi N, Kobayashi M, Kojima $\mathrm{H}$, et al. Phase II trial of paclitaxel and cisplatin as neoadjuvant chemotherapy for locally advanced gastric cancer. Cancer Chemother Pharmacol. 2013;71:1309-14.

16. Kosaka T, Akiyama H, Makino H, Takagawa R, Kimura J, Ono H, et al. Preoperative S-1 and docetaxel combination chemotherapy in patients with locally advanced gastric cancer. Cancer Chemother Pharmacol. 2014;73:281-5.

17. Oki E, Emi Y, Kusumoto T, Sakaguchi Y, Yamamoto M, Sadanaga $\mathrm{N}$, et al. Phase II study of docetaxel and S-1 (DS) as neoadjuvant 
chemotherapy for clinical stage III resectable gastric cancer. Ann Surg Oncol. 2014;21:2340-6.

18. Eto K, Hiki N, Kumagai K, Shoji Y, Tsuda Y, Kano Y, et al. Prophylactic effect of neoadjuvant chemotherapy in gastric cancer patients with postoperative complications. Gastric Cancer. 2018;21(4):703-9. https://doi.org/10.1007/s10120-017-0781-y Epub 2017 Nov 29.

19. Iwasaki Y, Terashima M, Mizusawa J, Katayama H, Nakamura $\mathrm{K}$, Katai H, et al. Randomized phase III trial of gastrectomy with or without neoadjuvant S-1 plus cisplatin for type 4 or large type 3 gastric cancer: Japan clinical oncology group study (JCOG0501). J Clin Oncol. 2018;15(15_suppl):4046-4046. https ://doi.org/10.1200/JCO.2018.36.15_suppl.4046

20. Japanese Gastric Cancer Association. Japanese classification of gastric carcinoma: 3rd english edition. Gastric Cancer. 2011;14:101-12. https://doi.org/10.1007/s10120-011-0041-5.

21. Dindo D, Demartines N, Clavien PA. Classification of surgical complications: a new proposal with evaluation in a cohort of 6336 patients and results of a survey. Ann Surg. 2004;240:205-13.

22. Katayama H, Kurokawa Y, Nakamura K, Ito H, Kanemitsu Y, Masuda N, et al. Extended Clavien-Dindo classification of surgical complications: Japan clinical oncology group postoperative complications criteria. Surg Today. 2016;46(6):668-85. https:// doi.org/10.1007/s00595-015-1236-x Epub 2015 Aug 20.

23. Lowy AM, Mansfield PF, Leach SD, Pazdur R, Dumas P, Ajani JA. Response to neoadjuvant chemotherapy best predicts survival after curative resection of gastric cancer. Ann Surg. 1999;229(3):303-8.

24. Nakamura K, Kuwata T, Shimoda T, Mizusawa J, Katayama H, Kushima R, et al. Determination of the optimal cutoff percentage of residual tumors to define the pathological response rate for gastric cancer treated with preoperative therapy (JCOG1004-A). Gastric Cancer. 2015;18(3):597-604. https://doi.org/10.1007/ s10120-014-0401-z Epub 2014 Jun 27.

25. Tsuburaya A, Mizusawa J, Tanaka Y, Fukushima N, Nashimoto A, Sasako M, et al. Neoadjuvant chemotherapy with S-1 and cisplatin followed by D2 gastrectomy with para-aortic lymph node dissection for gastric cancer with extensive lymph node metastasis. Br J Surg. 2014;101(6):653-60. https://doi.org/10.1002/bjs.9484 Epub 2014 Mar 25.

26. Zingoni A, Fionda C, Borrelli C, Cippitelli M, Santoni A, Soriani A. Natural killer cell response to chemotherapy-stressed cancer cells: role in tumor immunosurveillance. Front Immunol. 2017;8:1194. https://doi.org/10.3389/fimmu.2017.01194 eCollection2017.

Publisher's Note Springer Nature remains neutral with regard to jurisdictional claims in published maps and institutional affiliations. 